\title{
Fibrous hamartoma of infancy: A case report with typical ultrasonographic findings
}

\author{
Duygu Demirtaş-Güner ${ }^{1}$, Hilal Susam-Şen², Zuhal Akçören³, Bilgehan Yalçın², Berna \\ Oğuz ${ }^{4}$, Canan Akyüz ${ }^{2}$ \\ ${ }^{2}$ Division of Pediatric Oncology, and ${ }^{3}$ Pediatric Pathology, ${ }^{1}$ Department of Pediatrics, ${ }^{4}$ Department of Radiology, Hacettepe \\ University Faculty of Medicine, Ankara, Turkey. E-mail: duygudemirtas@gmail.com \\ Received: 5th April 2016, Revised: 31st May 2016, Accepted: 15th August 2016
}

\begin{abstract}
SUMMARY: Demirtaş-Güner D, Susam-Şen H, Akçören Z, Yalçın B, Oğuz B, Akyüz C. Fibrous hamartoma of infancy: A rare cause of axillary masses in children. Turk J Pediatr 2016; 58: 683-686.

Fibrous hamartoma of infancy (FHI) is a rare, benign lesion which is commonly seen under two years of age. Clinical and radiological features of FHI can mimic malignant soft tissue sarcomas, it is important to make differential diagnosis. Ultrasound (US) examination reveals heterogeneous echogenicity that can be also suggestive for other soft tissue tumors but newly defined "serpentine pattern" of intervening hypoechoic portions in the hyperechoic mass with poorly defined margins and with poor vascularity is special for FHI. Here we report a 15-month-old-boy with FHI with serpentine pattern on US. He initially presented with a painless mass in his left axilla existing for approximately seven months. The mass was successfully excised and he has been followed for three years without any evidence of recurrence. Fibrous hamartoma of infancy should always be considered in differential diagnosis in children under two years of age with a firm and solitary mass in the axilla especially when US reveals serpentine pattern with poorly defined margins and with poor vascularity. If these clinical and ultrasonographic findings are seen in a child under two years old, surgery can be performed without any additional imaging modalities. Awareness and careful assessment are important in order not to misdiagnose this benign mass for which surgical excision is curative.
\end{abstract}

Key words: fibrous hamartoma of infancy, ultrasonography, serpentine pattern, axillary mass.

Fibrous hamartoma of infancy (FHI) is a rare, completely benign lesion derived from fibroblasts and myofibroblasts of subcutaneous tissue ${ }^{1}$. Lesions are usually detected under two years of age, $15-20 \%$ of FHI are present at birth. Boys are more commonly affected than girls. FHI is usually presented with a solitary, firm, rapidly growing asymptomatic mass; mostly located in the axilla, shoulder and upper $\operatorname{arm}^{2}$.

Clinical and radiological features of FHI can mimic malignant soft tissue sarcomas. Ultrasound (US) examination reveals heterogeneous echogenicity that can also be suggestive for other soft tissue tumors but newly defined "serpentine pattern" of intervening hypoechoic portions in the hyperechoic mass with poorly defined margins and with poor vascularity is specific for $\mathrm{FHI}^{3}$. Definitive diagnosis can be achieved by histological evaluation. Local excision is treatment of choice; recurrence rate is less than $15 \%$, even if subtotal resection is performed.

Herein, we report a 15-month-old-boy with FHI with serpentine pattern on US and discuss the approach and differential diagnosis in an infant with an asymptomatic axillary mass.

\section{Case Report}

A 15-month-old boy born out of a nonconsanguineous marriage was admitted to Hacettepe University Children's Hospital, Division of Pediatric Oncology for the evaluation of the mass in his left axilla existing 
approximately for seven months. The mass did not cause any discomfort or pain to the patient. About one month ago before admission to our clinic, an US was done for the evaluation of the mass in another clinic. The US revealed a heterogeneous nodular lesion with poorly defined margins measuring $2.9 \times 1.6 \mathrm{~cm}$ in diameter. Enlargement of a regional lymph node due to Bacillus Calmette-Guérin (BCG) vaccine (the patient was vaccinated against tuberculosis when he was two months old) was suspected and follow-up was recommended.

However, the patient was admitted to our clinic due to enlargement in the mass. His birth and family history were unremarkable. The physical examination revealed a $3 \times 3$ $\mathrm{cm}$ firm, painless mass in his left axilla. Examination of other systems were normal. Investigations revealed normal complete blood count $(\mathrm{CBC})$ and erythrocyte sedimentation rate (ESR) with serum lactate dehydrogenase (LDH) level of $785 \mathrm{IU} / \mathrm{L}$ (normal <344). The purified protein derivative (PPD) skin test was $<10 \mathrm{~mm}$. Serological tests for EpsteinBarr virus and cytomegalovirus were negative and the chest X-ray was normal. The US revealed a heterogeneous solid lesion with both hypoechoic and hyperechoic portions with poorly defined margins measuring $3 \times 1.5 \mathrm{~cm}$ in diameter. The lesion was hypovascular on Doppler US. The lesion measuring $4 \times 4 \times 2.5 \mathrm{~cm}$ in size was surgically excised. On gross sections; poorly circumscribed, gray-white and nodular lesion was seen in the fat tissue. Smooth muscle actin was positive in dense fibrous areas, and vimentin was positive in both dense fibrous

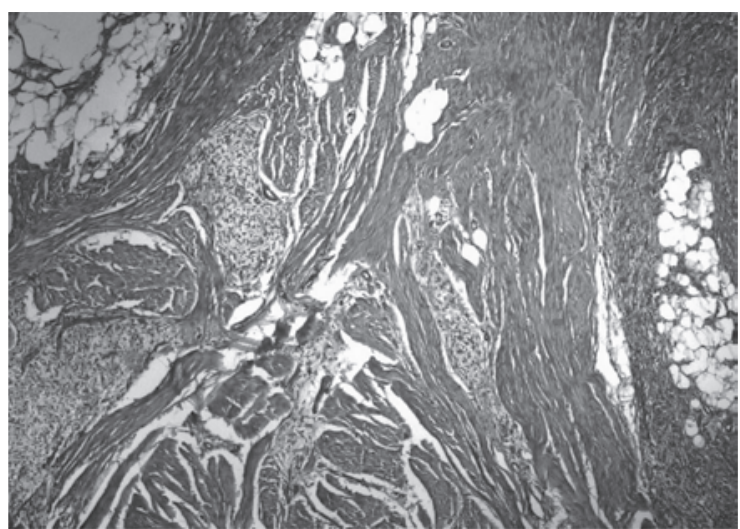

Fig 1. Mature adipose tissue, fibrous tissue and immature mesenchymal cells, consistent with fibrous hamartoma of infancy. areas and undifferentiated mesenchymal tissue. Histopathological examination was consistent with FHI (Fig. 1). Postoperatively, he has been followed for three years without any evidence of recurrence.

\section{Discussion}

At the outset, lymphadenopathy and tumor were the most possible causes due to presentation of our patient with a painless mass in the axilla. We did not think infectious diseases except nontuberculous mycobacterial lymphadenitis (NML), as cat scratch disease and pyogenic adenitis or suppurative BCG lymphadenitis primarily due to absence of fever, tenderness and other constitutional symptoms.

Non-suppurative BCG lymphadenitis which is presented as isolated enlarged axillary (rarely supraclavicular or cervical) lymph nodes ipsilateral to the site of BCG vaccination, usually resolves spontaneously over a few weeks to months without sequela ${ }^{4,5,6}$. Turnbull et al. ${ }^{7}$ reported that the onset of lymphadenitis occurred at a median of 63 days (range, 16-87 days) after BCG vaccination. Although location of the mass of our patient was compatible with the non-suppurative BCG lymphadenitis; onset and duration were not appropriate with regional lymphadenopathy due to BCG vaccine as it was perceived in the first clinic that he was admitted.

Nontuberculous mycobacterial lymphadenitis, typically seen in children between one and five years of age $\mathrm{e}^{7,8}$, is presented most commonly with unilateral, non-tender lymphadenitis. Although the cervicofacial nodes, particularly the submandibular nodes, are most frequently involved 7,8 , axillary lymphadenitis is also reported $^{8}$. Children with NML, usually have normal $\mathrm{CBC}$ and $\mathrm{ESR}^{8}$ and normal chest radiograph ${ }^{10}$ as our patient had. Erythema in $73.4 \%$ and discoloration of the skin in $15.2 \%$ of the patients were reported in the largest study of NML infections in children published to date $^{9}$, which were absent in our patient. There was a slight possibility that NML could be the definitive diagnosis, but FHI was diagnosed by histopathological evaluation.

Our most possible diagnosis was "soft tissue tumor" especially FHI on the basis of clinical and ultrasonographic findings. 
Soft tissue tumors are rare and heterogeneous group of tumors classified in accordance with histology, age and biological behavior ${ }^{11}$. Differential diagnoses between malignant and benign lesions are important to select the appropriate treatment. FHI is a benign and rare soft tissue lesion with typical histologic appearance characterized by a mixture of three components including fibrous tissue, primitive mesenchyme and adipose tissue. FHI was first reported by Reye ${ }^{12}$ in 1956 as subdermal fibromatous tumor of infancy and the current term was described by Enzinger ${ }^{2}$ in 1965. Although FHI is mostly located in the axilla, shoulder and upper arm, it might also be seen in the urogenital area, lumbar area, abdominal wall, scalp and face as described in the literature ${ }^{13,14,15}$. Affected children are healthy and no association with other malignancies is seen ${ }^{16}$. Familial predisposition is not reported ${ }^{2}$. FHI is more common in boys like other fibrous tumors of childhood as in our case. The tumoral lesions are usually presented as rapidly growing painless masses, usually $<5$ $\mathrm{cm}$ in size. Ultrasound examination reveals heterogeneous echogenicity that can be also suggestive for other soft tissue tumors ${ }^{17}$ but newly defined "serpentine pattern" with poorly defined margins and with poor vascularity is special for $\mathrm{FHI}^{3}$.

Our patient's US revealed serpentine pattern which was the typical ultrasonographic finding of FHI as discussed in the literature. So we considered FHI as the first differential diagnosis, and the mass was excised.

Metastasis has not been reported yet, but local recurrence has been reported after excision ${ }^{18}$. Total regression or malignant transformation has not been observed in the natural history of $\mathrm{FHI}^{16}$. Total local excision is the curative treatment of FHI. Even if radical complete resection is not possible anatomically, full recovery may be provided 19,20 . Chemotherapy or radiotherapy is not suggested in order to prevent overtreatment.

In conclusion, when a child under two years of age applies with a firm and solitary mass especially in the axilla and does not have fever, tenderness and other constitutional symptoms; FHI should be thought in the differential diagnosis, especially when US reveals newly defined serpentine pattern with poorly defined margins and with poor vascularity. If these clinical and ultrasonographic findings are seen in a child under two years old, surgery can be performed without any additional imaging modalities as MRI.

This case is reported to remind FHI as a clinically important entity in the differential diagnosis of masses of underarm which are often misdiagnosed as lymphadenopathy or other soft tissue tumors.

\section{REFERENCES}

1. Coffin CM, Dehner LP. Fibroblastic and myofibroblastic tumors in children and adolescents: a clinicopathologic study of 108 examples in 103 patients. Pediatr Pathol 1991; 11: 569-588.

2. Enzinger FM. Fibrous hamartoma of infancy. Cancer 1965; 18: 241-248.

3. Lee S, Choi YH, Cheon JE, Kim MJ, Lee MJ, Koh MJ. Ultrasonographic features of fibrous hamartoma of infancy. Skeletal Radiol 2014; 43: 649-653.

4. Goraya JS, Virdi VS. Bacille Calmette-Guerin lymphadenitis. Postgrad Med J 2002; 78: 327-329.

5. Riordan A, Cole T, Broomfield C. Fifteen-minute consultation: Bacillus Calmette-Guerin abscess and lymphadenitis. Arch Dis Child Educ Pract Ed 2014; 99: 87-89.

6. Venkataraman A, Yusuff M, Liebeschuetz S, Riddell A, Prendergast AJ. Management and outcome of Bacille Calmette-Guerin vaccine adverse reactions. Vaccine 2015; 33: 5470-5474.

7. Turnbull FM, McIntyre PB, Achat HM, et al. National study of adverse reactions after vaccination with Bacille Calmette-Guerin. Clin Infect Dis 2002; 34: 447-453.

8. Wolinsky E. Mycobacterial lymphadenitis in children: a prospective study of 105 nontuberculous cases with long-term follow-up. Clin Infect Dis 1995; 20: 954-963.

9. Tebruegge M, Pantazidou A, MacGregor D, et al. Nontuberculous mycobacterial disease in childrenepidemiology, diagnosis\&management at a tertiary center. PLoS One 2016; 11: e0147513.

10. Griffith DE, Aksamit T, Brown-Elliott BA, et al. An official ATS/IDSA statement: diagnosis, treatment, and prevention of nontuberculous mycobacterial diseases. Am J Respir Crit Care Med 2007; 175: 367-416.

11. Hashimoto H. Fibrous hamartoma of infancy. In Fletcher CDM, Unni KK, Mertens F (eds). World Health Organization Classification of Tumours. Pathology and Genetics of Tumours of the Soft Tissue and Bone. Lyon: IARC Press, 2002: 58-59.

12. Reye RD. A consideration of certain subdermal fibromatous tumours of infancy. J Pathol Bacteriol 1956; 72: 149-154.

13. Agrawal L, Bansal R, Singh J, Sharma S. Fibrous hamartoma of infancy in an unusual location- a case report. Gulf J Oncolog 2010; 8: 52-54. 
14. Seguier-Lipszyc E, Hermann G, Kaplinski C, Lotan G. Fibrous hamartoma of infancy. J Pediatr Surg 2011; 46: 753-755.

15. Sengar M, Mohta A, Manchanda V, Khurana N. Paratesticular fibrous hamartoma in an infant. Singapore Med J 2012; 53: 63-65.

16. Dickey GE, Sotelo-Avila C. Fibrous hamartoma of infancy: current review. Pediatr Dev Pathol 1999; 2: 236-243.

17. Eich GF, Hoeffel JC, Tschappeler H, Gassner I, Willi UV. Fibrous tumours in children: imaging features of a heterogeneous group of disorders. Pediatr Radiol 1998; 28: 500-509.
18. Miyamoto M, Tsunoda R, Gembun Y, et al. Recurrence of fibrous hamartoma of infancy excised 14 years after the primary surgery. J Neurosurg Pediatr 2010; 5: 136-139.

19. Carretto E, Dall'Igna P, Alaggio R, et al. Fibrous hamartoma of infancy: an Italian multi-institutional experience. J Am Acad Dermatol 2006; 54: 800-803.

20. Kang G, Suh YL, Han J, Kwon GY, Lee SK, Seo JM Fibrous hamartoma of infancy: an experience of a single institute. J Korean Surg Soc 2011; 81: 61-65. 